\title{
Avaliação da capacidade funcional dos idosos e fatores associados à incapacidade
}

\author{
Evaluation of the functional capacity of the elderly \\ and factors associated with disability
}

Bruno Rossi Barbosa ${ }^{1}$

Joyce Marques de Almeida ${ }^{2}$

Mirna Rossi Barbosa ${ }^{3}$

Luiza Augusta Rosa Rossi-Barbosa ${ }^{2}$

${ }^{1}$ Estratégia Saúde da Família, Prefeitura Municipal de Montes Claros. Av. Cula Mangabeira 211, Centro. 39.401-002 Montes Claros MG Brasil. rossifmc@yahoo.com.br

${ }^{2}$ Centro de Ciências da Saúde, Universidade

Estadual de Montes Claros.

${ }^{3}$ Policlínica Doutor Carlos

José do Espírito Santo,

Secretaria Municipal de

Saúde de Montes Claros.

\begin{abstract}
The scope of this study was to evaluate the functional capacity and analyze the characteristics associated with the disability of elderly individuals attended in a Family Health Strategy Unit in Montes Claros in the state of Minas Gerais. The Lawton and Brody scale and the Katz scale were used and then analyzed using multinomial logistic regression. 286 elderly individuals were interviewed with ages ranging from 60 to 103 years and a mean age of 71.2 years ( $S D \pm 8.3)$. Of these, $61.9 \%$ self-reported their independence in terms of functional capacity. The dependence only in IADL was positively associated with age $\geq 75$ years $(O R=$ 8.38), female gender $(\mathrm{OR}=3.64)$ and heart disease $(O R=3.24)$. The dependence in IADL and $A D L$ was positively associated with age $\geq 75$ years (OR $=6.40)$, without a partner $(O R=3.26)$, those who had had a stroke $(O R=51.85)$, heart disease (OR $=4.18)$ and diabetes $(O R=3.35)$. The highest proportion of elderly was considered independent to carry out basic and instrumental activities of daily living. However, a significant portion still has impaired functional status. It is essential that the teams of the Family Health Strategy strive for health promotion and disease prevention, and therapeutic interventions that can minimize the factors that influence functional capacity.

Key words Aged, Frail elderly individuals, Geriatrics, Public health
\end{abstract}

Resumo O objetivo deste estudo foi avaliar a capacidade funcional e analisar as características associadas à incapacidade dos idosos atendidos em uma Estratégia da Saúde da Família em Montes Claros-MG. Utilizou-se a escala de Lawton e Brody e a escala de Katz, analisadas pela regressão logística multinomial. Foram entrevistados 286 idosos cuja idade variou de 60 a 103 anos, média de 71,2 anos (DP $\pm 8,3)$. Destes, $61,9 \%$ autorreferiram independentes quanto à capacidade funcional. A dependência apenas na AIVD foi positivamente associada à faixa etária $\geq 75$ anos ( $O R=8,38)$, ao sexo feminino $(O R=3,64)$ e com doença cardíaca $(\mathrm{OR}=3,24)$. A dependência tanto nas AIVD como nas ABVD foi positivamente associada à faixa etária $\geq 75$ anos $(O R=6,40)$, sem companheiro $(O R=3,26)$, aos que apresentavam AVE $(O R=$ $51,85)$, doença cardíaca $(O R=4,18)$ e aos com diabetes $(O R=3,35)$. A maior proporção de idosos foi considerada independente para a realização de atividades básicas e instrumentais de vida diária. Contudo, uma parcela expressiva ainda apresenta comprometimento do estado funcional. É essencial que as equipes da Estratégia da Saúde da Família busquem a promoção da saúde e a prevenção de doenças, além de intervenções terapêuticas que possam minimizar os fatores que interferem na capacidade funcional.

Palavras-chave Idoso, Idoso com deficiência funcional, Gerontologia, Saúde coletiva 


\section{Introdução}

O crescimento da população de idosos é um fenômeno mundial e está ocorrendo em nível sem precedentes. Os dados do censo de 2010 do Instituto Brasileiro de Geografia e Estatística (IBGE) apontam para uma população de 190.732.694 pessoas, sendo que os idosos acima de 60 anos formam o grupo que mais cresceu na última década, representando $12,1 \%$ da população brasileira. Ressalta-se que os avanços da Medicina e as mudanças socioeconômicas têm elevado a média da expectativa de vida ao nascer de 45,5 anos, em 1940, para 74,1 anos, em 2011. Em relação ao gênero, os homens apresentam expectativa de 70,6 anos, enquanto as mulheres de 77,7 anos ${ }^{1}$.

De acordo com a Organização Mundial da Saúde (OMS), são consideradas idosas as pessoas com mais de 65 anos. Este referencial, entretanto, é válido para habitantes de países desenvolvidos. Nos países em desenvolvimento, como o Brasil, a terceira idade começa aos 60 anos $^{2}$. Esse segmento da população, pelo próprio processo de envelhecimento, apresenta maior prevalência de doenças crônico-degenerativas ${ }^{3}$.

Acrescenta-se o fato de que com o avançar da idade ocorrem mudanças morfológicas, funcionais e bioquímicas que abrangem todo o organismo ${ }^{4}$ e determinam a perda progressiva da capacidade de adaptação do indivíduo ao meio ambiente, ocasionando maior vulnerabilidade ${ }^{5}$.

O atual conceito de saúde engloba não meramente a ausência de doença, mas um estado de completo bem-estar físico, mental e social ${ }^{6}$. Requer, dessa forma, uma abordagem holística de qualquer que seja o paciente. E acompanhando essa evolução surgem no Brasil as Estratégias de Saúde da Família - ESF, com evidente papel de instrumento de reorganização e reestruturação do sistema público de saúde ${ }^{7}$.

Nesse contexto, a avaliação dos idosos nas ESF, grupo etário que possui grande demanda nesses serviços, deve incluir não só o diagnóstico das moléstias, mas também uma compreensão quanto aos aspectos funcionais. Estes envolvem, além da saúde física e mental, as condições sócio-econômicas e de capacidade de autocuidado, as quais irão revelar o grau de independência funcional do idoso. A avaliação da capacidade funcional torna-se, portanto, essencial para a escolha do melhor tipo de intervenção e monitorização do estado clínico-funcional dos idosos ${ }^{8}$.

Capacidade funcional pode ser definida como o potencial que os idosos apresentam para decidir e atuar em suas vidas de forma independente, no seu cotidiano'. E a incapacidade funcional refere-se à dificuldade ou necessidade de ajuda para o indivíduo executar tarefas no seu dia-a-dia ${ }^{10}$, abrangendo dois tipos de atividades: Atividades Básicas de Vida Diária (ABVD) e Atividades Instrumentais de Vida Diária (AIVD). A divisão em ABVD e AIVD começou com os estudos de Mahoney e Barthe ${ }^{11}$ e Lawton e Brody ${ }^{12}$. Os autores apresentaram duas escalas de avaliação funcional que classificavam as atividades cotidianas de acordo com o seu nível de complexidade. Lawton e Brody ${ }^{12}$ elaboraram uma escala para avaliar AIVD com oito atividades: preparar refeições, fazer tarefas domésticas, lavar roupas, manusear dinheiro, usar o telefone, tomar medicações, fazer compras e utilizar os meios de transporte.

O Índice de Katz é um dos instrumentos utilizados para avaliar as ABVD e foi criado por Sidney Katz, em 1963, para avaliar a capacidade funcional do indivíduo idoso ${ }^{13}$. Katz estabeleceu uma lista de seis itens que são hierarquicamente relacionados e refletem os padrões de desenvolvimento infantil, ou seja, que a perda da função no idoso começa pelas atividades mais complexas, como vestir-se, banhar-se, até chegar as de autorregulação como alimentar-se e as de eliminação ou excreção. Portanto, baseia-se na premissa de que o declínio funcional e a perda da capacidade para executar as atividades da vida diária nos pacientes idosos seguem um mesmo padrão de evolução, ou seja, perde-se primeiro a capacidade para banhar-se e, a seguir, para vestir-se, transferir-se da cadeira para a cama (e vice-versa) e alimentar-se. A recuperação dá-se na ordem inversa ${ }^{13}$.

O envelhecimento é um processo multifatorial e se manifesta de maneira peculiar e individual considerando as diferentes regiões geográficas ${ }^{14}$. Portanto, para promover um envelhecimento saudável, é necessário que cada equipe de saúde da família conheça o perfil da população idosa de sua região. Tal conhecimento fornecerá os subsídios necessários para uma promoção integral da saúde desse seguimento populacional. Desta forma, o objetivo deste estudo foi avaliar a capacidade funcional e analisar as características associadas à incapacidade dos idosos atendidos pela equipe da E.S.F. Lourdes II, na cidade de Montes Claros (MG).

\section{Métodos}

Foi realizado um estudo epidemiológico transversal, descritivo e analítico de base populacional com idosos adscritos na E.S.F. Lourdes II da cidade de 
Montes Claros (MG). Esta população cadastrada na E.S.F. totalizou 329 indivíduos.

A variável dependente foi a capacidade funcional do idoso, definida como nenhuma dificuldade para realizar ABVD ou AIVD, dependentes apenas nas AIVD, dependentes nas AIVD e ABVD caso relatassem necessidade de ajuda em pelo menos umas das atividades de cada dimensão. Utilizouse a escala de Lawton e Brody ${ }^{12}$, para mensurar as Atividades Instrumentais de Vida Diária (AIVD) e a escala de $\mathrm{Katz}^{13}$, para mensurar as Atividades Básicas de Vida Diária (ABVD).

Para conhecer o perfil social, econômico, demográfico e de saúde dos idosos foi aplicado um questionário estruturado na forma de entrevista. O protocolo incluiu os seguintes itens que se constituíram nas variáveis independentes: sexo, idade, escolaridade, profissão, estado civil, religião, renda do idoso, tipo de moradia, cor da pele autorreferida e patologias autorreferidas.

A idade foi categorizada em: 60-69 anos, 70 a 79 anos e 80 anos ou mais; quanto ao nível educacional os idosos foram classificados em: sem escolaridade, um a quatro anos de estudo, cinco a oito anos de estudo, mais de oito anos de estudo; a renda do idoso foi categorizada em menos de um salário mínimo, um salário mínimo, que correspondia a R \$ 510,00 (quinhentos e dez reais), um a três salários mínimos exclusive, três a cinco salários mínimos exclusive, cinco ou mais salários mínimos; devido à diversidade de profissão, esta foi categorizada em braçal (aquela que prepondera um grande esforço físico) e não braçal; a aposentadoria foi dicotomizada em sim ou não, bem como a moradia em casa própria.

As doenças crônicas foram mensuradas com base nas respostas afirmativas autorreferidas relativas à presença de oito delas: hipertensão arterial (pressão alta), outras doenças cardiovasculares (ataque cardíaco, doença coronária, angina, insuficiência cardíaca, chagas, outros problemas cardíacos), diabetes mellitus, doenças pulmonares (asma, bronquite, enfisema), câncer (excluídos somente os tumores menores de pele), tireoideopatias (hiper ou hipotireoidismo), acidente vascular encefálico - AVE (derrame, trombose) e problemas reumatológicos e osteomusculares (artrite, reumatismo, osteoporose, artrose). A variável "patologias autorreferidas" foi analisada de duas formas: cada doença crônica com resposta sim ou não e como multimorbidade ( $\operatorname{sim} /$ não) considerando afirmativamente a presença de duas ou mais doenças crônicas ${ }^{15,16}$.

Quando o idoso possuía algum déficit cognitivo ou de memória foi requerido ao cuidador responder o questionário bem como a assinatura do termo de consentimento livre e esclarecido.

A coleta de dados foi realizada em nível domiciliar e na unidade da E.S.F., no período de fevereiro a junho de 2010. A entrevista foi realizada por dois profissionais e uma acadêmica da área da saúde. A equipe foi submetida a treinamento para aplicação do questionário e realização da entrevista.

Realizou-se a distribuição de frequências simples das variáveis de interesse do estudo e regressão logística multinomial para estimar os fatores associados à incapacidade funcional. As variáveis independentes foram dicotomizadas e aquelas que apresentaram $\mathrm{p}<0,25$ na análise bivariada foram selecionadas para o modelo final, como potenciais fatores de confusão e introduzidas no modelo múltiplo seguindo a ordem decrescente do nível descritivo. A categoria capacidade funcional independente foi definida como referência e um nível de significância de 5\% ( $\mathrm{p} \leq 0,05)$ foi usado para a construção de intervalos de confiança de 95\% (IC 95\%) para as odds ratio (OR). Os dados foram tabulados e analisados utilizando-se o programa estatístico PASW Statistics 18.

O projeto de investigação foi aprovado pelo Comitê de Ética em Pesquisa da Universidade Estadual de Montes Claros (Unimontes). Todos os participantes assinaram um termo de consentimento livre e esclarecido, de acordo com a Resolução 196/96 do Conselho Nacional de Saúde, relativa à pesquisa envolvendo seres humanos ${ }^{17}$.

\section{Resultados}

Dos 329 idosos cadastrados na E.S.F. Lourdes II, dois recusaram participar da pesquisa, seis haviam falecido e 35 se mudaram ou moram na zona rural, ou não foram encontrados em seu domicílio após três visitas em dias e horários diferentes.

Desta forma, foram entrevistados 286 idosos, o que equivale a $86 \%$ do total. A idade variou de 60 a 103 anos, sendo a média de 71,2 anos (DP $\pm 8,3)$ e a média da escolaridade foi de 3,2 anos $(\mathrm{DP} \pm 3,4)$. Os dados sócio-demográficos estão presentes na Tabela 1 .

Relativo às patologias autorreferidas, 135 $(47,2 \%)$ idosos alegaram sofrer de uma morbidade, $85(29,8 \%)$ de duas a três doenças, $63(22,0 \%)$ não apresentaram nenhuma doença, três (10\%) afirmaram conviver com quatro ou mais enfermidades. Entre as ocorrências de maior frequência, a Hipertensão Arterial Sistêmica foi referida por 148 (51,7\%) dos idosos. 
Tabela 1. Dados sociodemográficos dos idosos do ESF do bairro de Lourdes, Montes Claros-MG, 2010.

\begin{tabular}{|c|c|c|}
\hline Dados sociodemográficos & $\mathbf{n}$ & $\%$ \\
\hline \multicolumn{3}{|l|}{ Sexo } \\
\hline feminino & 181 & 63,3 \\
\hline masculino & 105 & 36,7 \\
\hline \multicolumn{3}{|l|}{ Faixa etária } \\
\hline 60 a 69 & 141 & 49,7 \\
\hline 70 a 79 & 91 & 32,0 \\
\hline 80 ou mais & 52 & 18,3 \\
\hline \multicolumn{3}{|l|}{ Estado civil } \\
\hline casados & 153 & 53,5 \\
\hline viúvos & 94 & 32,9 \\
\hline solteiros & 21 & 7,3 \\
\hline separados & 18 & 6,3 \\
\hline \multicolumn{3}{|l|}{ Religião } \\
\hline católicos & 222 & 77,6 \\
\hline evangélicos & 59 & 20,6 \\
\hline sem religião & 3 & 1,1 \\
\hline espírita & 2 & 0,7 \\
\hline \multicolumn{3}{|l|}{ Escolaridade } \\
\hline analfabeto & 80 & 28,4 \\
\hline a 4 anos de estudo & 136 & 48,2 \\
\hline a 8 anos de estudo & 44 & 15,6 \\
\hline de 8 anos de estudo & 22 & 7,8 \\
\hline \multicolumn{3}{|l|}{$\operatorname{Renda}^{*}$} \\
\hline$<$ que um salário mínimo & 50 & 17,7 \\
\hline um salário mínimo & 146 & 51,8 \\
\hline$>$ que um salário a três salários & 72 & 25,5 \\
\hline$>$ que três salários a cinco salários & 7 & 2,5 \\
\hline$>$ que cinco salários & 7 & 2,5 \\
\hline \multicolumn{3}{|l|}{ Profissão } \\
\hline braçal & 204 & 75,3 \\
\hline não braçal & 67 & 24,7 \\
\hline \multicolumn{3}{|l|}{ Aposentado } \\
\hline $\operatorname{sim}$ & 181 & 63,5 \\
\hline não & 104 & 36,5 \\
\hline \multicolumn{3}{|l|}{ Casa própria } \\
\hline $\operatorname{sim}$ & 267 & 94,0 \\
\hline não & 17 & 6,0 \\
\hline \multicolumn{3}{|l|}{ Cor da pele autorreferida } \\
\hline parda & 154 & 54,0 \\
\hline branca & 81 & 28,4 \\
\hline negra & 44 & 15,4 \\
\hline outras & 7 & 2,2 \\
\hline
\end{tabular}

Sobre a capacidade funcional, a Tabela 2 apresenta a frequência entre os idosos.

Quanto aos que apresentaram dependência na ABVD, 19 (6,6\%) eram dependentes para a minoria (até três) das ABVD e 16 (5,6\%) dependentes para a maioria destas atividades. Os idosos precisaram de ajuda principalmente para: controle da micção $(9,4 \%)$, vestir-se $(7,6 \%)$ e banhar-se $(6,6 \%)$.
Em relação às AIVD foram considerados tanto aqueles com dependência apenas em AIVD quanto os com dependência nas AIVD e ABVD e observou-se maior dependência para ir a lugares mais distantes sozinhos $(24,1 \%)$, lavar e passar roupa $(22,0 \%)$, usar o telefone $(18,9 \%)$ conforme Tabela 3.

As variáveis independentes: idade, alfabetização, sexo, doença cardíaca, estado civil, patologias, doença pulmonar, profissão, renda, diabetes, artropatia, casa própria e hipertensão obtiveram $\mathrm{p}<0,20$ e por isso foram incluídas no modelo

Tabela 2. Distribuição dos idosos do ESF do bairro de Lourdes segundo a capacidade funcional. Montes Claros-MG, 2010.

\begin{tabular}{lrc}
\hline \multicolumn{1}{r}{ Capacidade Funcional } & n & \% \\
\hline Independente & 177 & 61,9 \\
Dependente somente na AIVD & 74 & 25,9 \\
Dependente na ABVD e AIVD & 35 & 12,2 \\
Total & 286 & 100 \\
\hline
\end{tabular}

Tabela 3. Distribuição dos idosos do ESF Lourdes II segundo as incapacidades nas AIVD. Montes ClarosMG, 2010.

\begin{tabular}{lrr}
\hline Incapacidade nas AIVD & n & $\%$ \\
\hline $\begin{array}{l}\text { Dependências } \\
\text { ir a lugares mais distantes (transporte) }\end{array}$ & 74 & 25,9 \\
$\quad$ parcial & 40 & 14,0 \\
$\quad$ incapaz & 29 & 10,1 \\
lavar e passar roupa & & \\
$\quad$ ajuda parcial & 22 & 7,7 \\
$\quad$ incapaz & 41 & 14,3 \\
usar o telefone & & \\
$\quad$ ajuda parcial & 34 & 11,9 \\
$\quad$ incapaz & 20 & 7,0 \\
fazer compras & & \\
$\quad$ ajuda parcial & 20 & 7,0 \\
$\quad$ incapaz & 32 & 11,2 \\
arrumar a casa & & \\
$\quad$ ajuda parcial & 22 & 7,7 \\
$\quad$ incapaz & 29 & 10,1 \\
tomar seus remédios corretamente & & \\
$\quad$ ajuda parcial & 22 & 7,7 \\
$\quad$ incapaz & 19 & 6,6 \\
preparar refeições & & \\
$\quad$ ajuda parcial & 10 & 3,5 \\
$\quad$ incapaz & 29 & 10,1 \\
controlar seu dinheiro & & \\
$\quad$ ajuda parcial & 16 & 5,6 \\
$\quad$ incapaz & 21 & 7,3 \\
\hline$\quad$ & &
\end{tabular}


de regressão logística multinomial. Os resultados da análise ajustada se encontram na Tabela 4. A dependência apenas na AIVD foi positivamente associada à faixa etária $\geq 75$ anos $(\mathrm{OR}=8,38)$, ao sexo feminino $(\mathrm{OR}=3,64)$ e com doença cardíaca $(\mathrm{OR}=3,24)$. A dependência tanto nas AIVD, como nas $A B V D$, foi positivamente associada à faixa etária $\geq 75$ anos $(\mathrm{OR}=6,40)$, sem companheiro $(\mathrm{OR}=3,26)$, aos que apresentavam AVE $(\mathrm{OR}=$ $51,85)$, doença cardíaca $(\mathrm{OR}=4,18)$ e aos com diabetes $(\mathrm{OR}=3,35)$.

\section{Discussão}

O presente estudo permitiu avaliar a capacidade funcional dos idosos adscritos na E.S.F. do bairro de Lourdes da cidade de Montes Claros - MG. A frequência da incapacidade funcional foi menor que a registrada em outros estudos ${ }^{3,18-20}$. Com o aumento da expectativa de vida e com o avanço de estudos que buscam promover a qualidade de vida dos idosos, pode-se inferir que a prevalência de idosos mais ativos e independentes é cada vez maior.

Observou-se maior prevalência nas AIVD do que nas $\mathrm{ABVD}$, o que está de acordo com outras pesquisas $^{18-20}$. Hierarquicamente, as perdas ocorrem de atividades instrumentais de vida diária para atividades básicas de vida diária ${ }^{19}$, devido às AIVD exigirem maior integridade física e cognitiva comparada às $\mathrm{ABVD}^{18,20}$. Todavia, estudos apontam uma maior prevalência de dependência em AIVD em relação à presente pesquisa ${ }^{18,20,21}$.

Com relação à prevalência de dependência em ABVD, a literatura também registra diferenças entre os estudos. A França é o país europeu com maior dependência nas ABVD (28\%) e a Irlanda registra o menor índice ( $8 \%$ ) da população com mais de 65 anos $^{18}$. Na Ásia é possível encontrar menores taxas de incapacidade funcional. Em Singapura a dependência em pelo menos um tipo de ABVD é de 6,6\% $\%^{22}$. Estas discrepâncias provavelmente se devem às diferentes realidades socioeconômicas e culturais como também à metodologia utilizada ${ }^{18}$. Resultados semelhantes ao do presente estudo nas ABVD foram encontrados nas pesquisas realizadas na $\mathrm{Bahia}^{20}$ e São Paulo $^{19}$, sendo que nesta última a metodologia para a inclusão dos participantes foi diferente. Por outro lado, em dois estudos realizados na cidade de Goiânia - GO, a prevalência de idosos dependentes nas ABVD foi maior ${ }^{3,14}$.

A maior frequência de dependência em ABVD foi para a incontinência urinária, como encontrado em outros estudos ${ }^{3,18,20,23}$. Tal fato é preocupante podendo ser constrangedor e levar ao isolamento social ${ }^{3,14,24}$, a alterações na autoestima e autoimagem, influenciando nas atividades instrumentais de vida diária ${ }^{14}$.

A incontinência urinária é vista, erroneamente, como processo natural do envelhecimento pela população, no geral ${ }^{23}$ e, apesar da sua frequência

Tabela 4. Fatores associados à incapacidade funcional dos idosos do ESF Lourdes II. Montes Claros-MG, 2010.

\begin{tabular}{|c|c|c|c|c|c|c|}
\hline \multirow[b]{2}{*}{ Variáveis } & \multicolumn{3}{|c|}{ Dependência somente em AIVD } & \multicolumn{3}{|c|}{ Dependência em ABVD e AIVD } \\
\hline & p-valor & OR ajustada & IC (95\%) & p-valor & OR ajustada & IC (95\%) \\
\hline \multicolumn{7}{|l|}{ Faixa etária } \\
\hline$<75$ anos & & 1 & \multirow{2}{*}{$4,20-16,73$} & \multirow{2}{*}{$<0,001$} & 1 & \\
\hline$\geq 75$ anos & $<0,001$ & 8,38 & & & 6,40 & $2,62-15,62$ \\
\hline \multicolumn{7}{|l|}{ Sexo } \\
\hline Masculino & & 1 & \multirow{2}{*}{$1,67-7,92$} & \multirow{2}{*}{0,581} & 1 & \\
\hline Feminino & 0,001 & 3,64 & & & 0,76 & $0,29-2,00$ \\
\hline \multicolumn{7}{|l|}{ Estado civil } \\
\hline Com companheiro & & 1 & \multirow{2}{*}{$0,59-2,23$} & \multirow{2}{*}{0,016} & 1 & \\
\hline Sem companheiro & 0,678 & 1,15 & & & 3,26 & $1,25-8,51$ \\
\hline \multicolumn{7}{|l|}{ AVE } \\
\hline Não & & 1 & \multirow{2}{*}{$0,93-99,78$} & \multirow{2}{*}{0,001} & 1 & \\
\hline Sim & 0,057 & 9,65 & & & 51,85 & $5,35-502,46$ \\
\hline \multicolumn{7}{|l|}{ Doença cardíaca } \\
\hline Não & & 1 & \multirow{2}{*}{$1,07-9,83$} & \multirow{2}{*}{0,028} & 1 & \\
\hline Sim & 0,038 & 3,24 & & & 4,18 & $1,17-14,96$ \\
\hline \multicolumn{7}{|l|}{ Diabetes mellitus } \\
\hline Não & & 1 & \multirow{2}{*}{$0,93-5,79$} & \multirow{2}{*}{0,043} & 1 & \\
\hline Sim & 0,072 & 2,32 & & & 3,35 & $1,04-10,82$ \\
\hline
\end{tabular}


e das repercussões funcionais, é a queixa mais negligenciada no exame clínico usual. Sua presença deve ser prontamente reconhecida, solicitando-se ao paciente a descrição da sua micção (volume, frequência, intervalo, aspecto, etc.), dando-se ênfase na presença de urgência miccional e noctúria ${ }^{24}$.

O diagnóstico de incontinência urinária deve ser seguido por uma ampla investigação das possíveis causas ${ }^{24}$. Faz-se necessário planejar estratégias que melhorem o estilo de vida dos idosos com promoção, tratamento e reabilitação da capacidade funcional do esfíncter ${ }^{14}$. A promoção dever ser realizada por meio de mudanças comportamentais e exercícios que visam fortalecer o esfíncter uretral.

Ao analisar a dependência somente nas AIVD, a maior incapacidade referiu-se a ir a lugares mais distantes sozinhos, usando algum transporte, seguida de lavar e passar roupa e usar o telefone. $\mathrm{O}$ tipo de atividade em que os idosos apresentaram maior dependência é diversificado entre os estudos. Em Pelotas - $\mathrm{RS}^{23}$, realizar deslocamentos utilizando algum meio de transporte também foi a atividade em que os idosos apresentaram maior dependência. Em Lafaiete Coutinho - $\mathrm{BA}^{20} \mathrm{e}$ em Cuiabá - MT ${ }^{25}$, idosos tiveram mais dificuldade quanto ao uso do telefone. Em Goiânia - GO ${ }^{3}$, a maior incapacidade relatada foi para lavar e passar roupa. Em outro estudo realizado, também, em Goiânia - $\mathrm{GO}^{14}$, a proporção de idosos com dependência quanto ao manuseio de dinheiro e uso de meios de transporte foram superiores às demais atividades.

O modelo de regressão múltiplo mostrou que o grupo etário $\geq 75$ anos, do sexo feminino e a presença de doença cardíaca foram fatores associados à incapacidade funcional apenas nas AIVD. Quanto aos fatores associados à incapacidade funcional nas ABVD e AIVD, o presente estudo apontou que a dependência é maior no grupo etário $\geq 75$ anos, sem companheiro, que tiveram AVE, doença cardíaca e diabetes mellitus.

Outros estudos ${ }^{18,20,23,26}$ apontaram que com o avanço da idade, maior é a probabilidade de dependência tanto nas AIVD quanto nas ABVD. Isto porque a capacidade de realizar uma tarefa envolve a integração de múltiplos sistemas fisiológicos que com o avançar dos anos, gradualmente entram em declínio ${ }^{20}$. No estudo realizado em Belo Horizonte - $\mathrm{MG}^{27}$, o risco de adquirir dependência em ABVD foi maior entre idosos acima de 80 anos comparados aos de 60 a 69 anos. Na pesquisa realizada em São Carlos - SP ${ }^{19}$, verificou-se fortíssima correlação entre faixa etária e o índice de Katz, ou seja, conforme a faixa etária aumenta, o grau de dependência dos idosos também aumenta.
Os resultados em relação ao sexo indicam que as mulheres são normalmente mais dependentes nas AIVD que os homens ${ }^{18,25,28}$. Embora as mulheres sejam mais longevas apresentam pior qualidade de vida ${ }^{18,28}$. Estudo que utilizou outro instrumento para medição da capacidade funcional dos idosos também observou maior chance de incapacidade entre pessoas do sexo feminino ${ }^{29}$.

A incapacidade funcional é menor entre os casados, comparados aos sem companheiro ${ }^{18}$. No presente estudo, a categoria "sem companheiro" engloba os idosos solteiros, viúvos e divorciados, e apresentou maior possibilidade de dependência para ABVD e não para AIVD isoladamente. Em contrapartida, no estudo realizado em Porto Alegre $(\mathrm{RS})^{26}$, o estado civil permaneceu no modelo final apenas para AIVD, indicando que os idosos que não tinham companheiro eram mais dependentes nestas atividades que os casados.

Doenças crônicas constituem um fator de risco para incapacidade funcional de idosos ${ }^{30}$. Independente das demais variáveis, diabetes mellitus, AVE e doenças cardíacas tiveram associação com dependência em $A B V D$, sendo que esta última patologia apresentou associação, também, com dependência em AIVD.

A presença de doenças cardíacas influencia negativamente na capacidade funcional de ido$\operatorname{sos}^{31}$. Em outro estudo, cinco doenças crônicas, dentre elas, os problemas cardíacos, foram os maiores determinantes da incapacidade funcional dos idosos canadenses pesquisados ${ }^{32}$. A doença de Chagas foi a cardiopatia relatada por alguns dos idosos que autorreferiram quanto às doenças cardíacas. De acordo com o inquérito sorológico nacional realizado em 1975 a 1980, a presença de Triatoma infestans foi o principal vetor da enfermidade no Brasil, sendo o norte de Minas Gerais uma área com infestações maciças ${ }^{33}$. No estudo de Ribeirão Preto (SP) ${ }^{34}, 78,6 \%$ dos chagásicos eram dependentes em ABVD.

Em relação ao diabetes, 38\% de idosos com esta condição mostrou deterioração funcional ${ }^{35}$. Em estudo realizado nos Estados Unidos, idosos com diabetes apresentaram prevalência de 37,2\% de incapacidade funcional para ABVD e de 43,6\% para $\mathrm{AIVD}^{36}$. O indivíduo acometido pelo diabetes mellitus precisa adotar medidas de autocuidado e de controle ao longo de sua vida, pois a mesma ainda não é passível de cura até a atualidade ${ }^{37}, \mathrm{o}$ que traz efeitos negativos significativos para sua qualidade de vida ${ }^{38}$.

Estudo que utilizou outro modelo de avaliação da capacidade funcional de idosos observou que diabetes e AVE apresentaram associações com in- 
capacidade funcional grave ${ }^{27}$. No presente estudo, o risco de apresentar dependência em ABVD é 51 vezes entre aqueles que tiveram AVE, comparados aos que não tiveram esta doença. O AVE pode causar ou exacerbar problemas associados com a dependência funcional, tais como, disfunção cognitiva, depressão, distúrbio de comunicação, alteração da marcha, incontinência urinária, entre outras, e em alguns casos, tem sido relatado com a principal causa de incapacidade funcional. É importante notar, que esta é uma doença evitável, com bom controle dos fatores de risco cardiovasculares ${ }^{39}$.

A probabilidade de apresentar incapacidade funcional pode aumentar em função do número de morbidades ${ }^{40,41}$. Entretanto, esta variável não permaneceu no modelo ajustado da presente pesquisa.

A capacidade funcional influencia na qualidade de vida dos idosos ${ }^{42}$. Neste sentido, é dever das equipes de E.S.F. identificar dados referentes à fragilidade dos idosos e da atenção à saúde dos mesmos, além de seus pontos passíveis de intervenção para eliminação de fatores de risco relacionados com a capacidade funcional.

Os resultados desta pesquisa devem ser considerados à luz de algumas limitações. Por ter um desenho metodológico transversal, não coube a este estudo buscar explicações para as associações encontradas. É necessário que sejam realizados estudos longitudinais para se investigar mais detalhadamente as relações entre os fatores associados e a incapacidade funcional e, consequentemente, desenvolver estratégias de promoção da saúde e prevenção de doenças nesta população.

\section{Conclusão}

A maior proporção de idosos pertencentes a essa E.S.F. foi considerada independente para a realização de atividades básicas e instrumentais de vida diária. Contudo, uma parcela expressiva ainda apresenta comprometimento do estado funcional, podendo acarretar em prejuízos físicos, psicológicos e sociais.

A incapacidade funcional é mais prevalente em idosos mais velhos, do sexo feminino e naqueles que não possuem companheiro. A presença de doenças como AVE, diabetes mellitus e doenças cardíacas também influenciam na capacidade funcional dos idosos.

Com o aumento da longevidade, é essencial que as equipes de ESF busquem a promoção da saúde e a prevenção de doenças, além de intervenções terapêuticas que possam minimizar os fatores que interferem na capacidade funcional, promovendo maior autonomia e qualidade de vida a este seguimento populacional.

\section{Colaboradores}

BR Barbosa participou do projeto, coleta de dados e redação do artigo. JM Almeida participou da coleta de dados, tabulação e redação do artigo. MR Barbosa e LAR Rossi-Barbosa participaram da análise, interpretação dos dados e redação do artigo.

\section{Agradecimentos}

Ao Programa Institucional de Bolsas de Iniciação Científica da Universidade Estadual de Montes Claros-BIC/UNI. 


\section{Referências}

1. Instituto Brasileiro de Geografia e Estatística (IBGE). Senso 2010. Rio de Janeiro: IBGE; 2010.

2. Zimerman GI. Velhice. Aspectos biopsicossociais. Porto Alegre: Artes Médicas Sul; 2007.

3. Nakatani AYK, Silva LB, Bachion MM, Nunes DP. Capacidade funcional em idosos na comunidade e propostas de intervenções pela equipe de saúde. Rev Eletrônica Enferm [periódico na Internet] 2009 [acessado 2010 jun 14]; 11(1):144-150. Disponível em: http://www.fen.ufg. br/revista/v11/n1/pdf/v11n1a18.pdf

4. Guimarães LHCT, Galdino DCA, Martins FLM, Abreu SR, Lima M, Vitorino DFM. Avaliação da Capacidade Funcional de Idosos em Tratamento Fisioterapêutico. Revista Neurociências 2004; 12(3).

5. Carvalho Filho ET, Papaleo Netto M. Geriatria: fundamentos, clínica e terapêutica. São Paulo: Atheneu; 2000.

6. United Nations (UN). Office for the Coordination of Humanitarian Affairs (ReliefWeb). Glossary of humanitarian terms. UN: Geneva; 2008.

7. Viana ALA, Poz MRD. A reforma do sistema de saúde no Brasil e o Programa de saúde da família. Physis: Rev Saúde Coletiva 2005; 15(Supl.):225-264.

8. Ricci NA, Kubota MT, Cordeiro RC. Concordância de observações sobre a capacidade funcional de idosos em assistência domiciliar. Rev Saude Publica 2005; 39(4):655-662.

9. Fiedler MM, Peres KG. Capacidade funcional e fatores associados em idosos do sul do Brasil: um estudo de base populacional. Cad Saude Publica 2008; 24(2):409-415.

10. Alves LC, Leite IC, Machado CJ. Conceituando e mensurando a incapacidade funcional da população idosa: uma revisão de literatura. Cienc Saude Colet 2008; 13(4):1199-1207.

11. Mahoney FI, Barthel D. Functional evaluation: the Barthel Index. Md. State Med J. 1965; 14:61-65.

12. Lawton MP, Brody EM. Assessment of older people: self-maintaining and instrumental activities of daily living. Gerontologist 1969; 9:179-186.

13. Katz S, Ford AB, Moskowitz RW, Jackson BA, Jaffe MW. Studies of illness in the aged. The index of ADL: a standardized measure of biological and psychosocial function. JAMA 1963; 185(12):914-919.

14. Costa EC, Nakatani AYK, Bachion MM. Capacidade de idosos da comunidade para desenvolver atividades de vida diária e atividades instrumentais de vida diária. Acta Paul. Enfem 2006; 19(1):43-48.

15. Van Den Akker M, Buntinx F, Roos S, Knottnerus JA. Comorbidity or multimorbidity: what's in a name? A review of the literature. Eur J Gen Pract 1996; 2(2):65-70.

16. Ribeiro KT. Fatores associados à qualidade de vida relacionada à saúde de idosos residentes no município de São Paulo - Estudo SABE: Saúde, Bem estar e Envelhecimento [tese]. São Paulo: Faculdade de Saúde Pública; 2011.

17. Brasil. Ministério da Saúde (MS). Conselho Nacional de Saúde. Resolução no. 196 de 10 de outubro de 1996. Diretrizes e Normas Regulamentadoras de Pesquisas Envolvendo Seres Humanos. Diário Oficial da União 1996; 16 out.
18. Millán-Calenti JC, Tubío J, Pita-Fernández S, GonzálezAbraldes I, Lorenzo T, Fernández-Arruty T, Maseda A. Prevalence of functional disability in activities of daily living (ADL), instrumental activities of daily living (IADL) and associated factors, as predictors of morbidity and mortality. Arch Gerontol Geriatr 2010; 50(3):306-310.

19. Santos AA, Pavarini SC. Functionality of elderly people with cognitive impairments in different contexts of social vulnerability. Acta Paul Enferm 2011; 24(4):520-526

20. Freitas RS, Fernandes MH, Coqueiro RS, Reis Júnior WM, Rocha SV, Brito TA. Capacidade funcional e fatores associados em idosos: estudo populacional. Acta Paul Enferm 2012; 25(6):933-939.

21. Nunes DP, Nakatani AYK, Silveira EA, Bachion MM, Souza MR. Capacidade funcional, condições socioeconômicas e de saúde de idosos atendidos por equipes de Saúde da Família de Goiânia (GO, Brasil). Cien Saude Colet 2010; 15(6):2887-2898.

22. Ng TP, Niti M, Chiam PC, Kua EH. Prevalence and Correlates of Functional Disability in Multiethnic Elderly Singaporeans. J Am Geriatr Soc 2006; 54(1):21-29.

23. Del Duca GF, Silva MC, Hallal PC. Incapacidade funcional para atividades básicas e instrumentais da vida diária em idosos. Rev Saude Publica 2009; 43(5):796-805.

24. Moraes EN. Atenção à Saúde do Idoso: Aspectos Conceituais. Brasília: Organização Pan-Americana da Saúde; 2012.

25. Oliveira PH, Mattos IE. Prevalência e fatores associados à incapacidade funcional em idosos institucionalizados no Município de Cuiabá, Estado de Mato Grosso, Brasil, 2009-2010. Epidemiol Serv Saúde 2012; 21(3):395-406.

26. Pereira GN, Bastos GAN, Del Duca GF, Bós AJG. Indicadores demográficos e socioeconômicos associados à incapacidade funcional em idosos. Cad Saude Publica 2012; 28(11):2035-2042.

27. Giacomin KC, Peixoto SV, Uchoa E, Lima-Costa MF, Estudo de base populacional dos fatores associados à incapacidade funcional entre idosos na Região Metropolitana de Belo Horizonte, Minas Gerais, Brasil. Cad Saude Publica 2008; 24(6):1260-1270.

28. Alves LC, Leite IC, Machado CJ. Fatores associados à incapacidade funcional dos idosos no Brasil: análise multinível. Rev Saude Publica 2010; 44(3):468-478.

29. Santos MIPO, Griep RH. Capacidade Funcional de Idosos Atendidos em um programa do SUS em Belém. PA. Cien Saude Colet 2013; 18(3):753-761.

30. Rodrigues MAP, Facchini LA, Thume E, Maia F. Gender and incidence of functional disability in the elderly: a systematic review. Cad Saude Publica 2009; 25(Supl. 3):S464-S476.

31. Alves LC, Leimann BCQ, Vasconcelos MEL, Carvalho MS, Vasconcelos AGG, Fonseca TCO, Lebrão ML, Laurenti R. A influência das doenças crônicas na capacidade funcional dos idosos do Município de São Paulo, Brasil. Cad Saude Publica 2007; 23(8):1924-1930. 
32. Griffith L, Raina P, Wu H, Zhu B, Stathokostas L. Population attributable risk for functional disability associated with chronic conditions in Canadian older adults. Age Ageing 2010; 39(6):738-745.

33. Silveira AC, Silva GR, Prata A. O Inquérito de soroprevalência da infecção chagásica humana (1975-1980). Rev Soc Bras Med Trop 2011; 44(Supl. II):33-39.

34. Rodrigues RAP, Scudeller PG, Pedrazzi EC, Schiavetto FV, Lange C. Morbidade e sua interferência na capacidade funcional de idosos. Acta Paul Enferm 2008; 21(4):643-648.

35. Blaum CS, Ofstedal MB, Langa KM, Wray LA. Functional status and health outcomes in older americans with diabetes mellitus. J Am Geriatr Soc 2003; 51(6):745-753.

36. Kalyani RR, Saudek CD, Brancati FL, Selvin E. Association of diabetes, comorbidities, and $\mathrm{AlC}$ with functional disability in older adults: results from the National Health and Nutrition Examination Survey (NHANES), 1999-2006. Diabetes Care 2010; 33(5):1055-1060.

37. Fernandes MGM, Pereira MAP, Honorato MM, Fernandes BM. Indicadores Sociodemográficos e Capacidade Funcional de Idosos Diabéticos. Revista Brasileira de Ciências da Saúde 2011; 15(1):57-64.

38. Huang ES, Brown SE, Ewigman BG, Foley EC, Meltzer DO. Patient perceptions of quality of life with diabetes -related complications and treatments. Diabetes Care 2007; 30(10):2478-2483.

39. Barrantes-Monge M, García-Mayo EJ, Gutiérrez-Robledo LM, Miguel-Jaimes A. Dependencia funcional y enfermedades cronicas en ancianos mexicanos. Salud Publica Mex [revista en la Internet]. 2007; [acessado 2013 abr 17]; 49(Supl 4):S459-466. Disponible en: http:// www.scielo.org.mx/scielo.php?script = sci_arttext\&pid = S0036-36342007001000004\&lng $=\mathrm{es}$

40. Santos KA, Koszuoski R, Dias-da-Costa, JS, Pattussi MP. Fatores associados com a incapacidade funcional em idosos do município de Guatambu, Santa Catarina, Brasil. Cad Saude Publica 2007;23(11):2781-2788.

41. Marengoni A, Strauss E, Rizzuto D, Winblad B, Fratiglioni $\mathrm{L}$. The impact of chronic multimorbidity and disability on functional decline and survival in elderly persons. A community-based, longitudinal study. J Intern Med 2009; 265(2):288-295.

42. Paskulin L, Vianna L, Molzahn AE. Factors associated with quality of life of Brazilian older adults. Int Nurs Rev 2009; 56(1):109-115

Artigo apresentado em 15/03/2013

Aprovado em 29/04/2013

Versão final apresentada em 07/05/2013 
